\title{
El debate sobre el sistema de acreditación del profesorado en fisioterapia desde una perspectiva académica y científica
}

\author{
The debate on the teacher's accreditation system in physiotherapy from an \\ academic and scientific perspective
}

\author{
Sonia Souto Camba ${ }^{\mathrm{a}, \mathrm{b}}$, María del Carmen García Ríos ${ }^{\mathrm{a}, \mathrm{c}}$, Raquel Chillón \\ Martínez ${ }^{\mathrm{a}, \mathrm{d}}$, José Ríos Día ${ }^{\mathrm{a}, \mathrm{e}}$, Sergio Hernández Fernández ${ }^{\mathrm{a}, \mathrm{f}}$ y Ana María Martín \\ Nogueras $^{\mathrm{a}, \mathrm{g}}$
}

\footnotetext{
${ }^{a}$ Junta Permanente de la AEF-ES; ${ }^{b}$ Universidad de A Coruña, A Coruña: ${ }^{c}$ Universidad de Granada, Granada; ${ }^{d}$ Universidad de Sevilla; Sevilla ${ }^{\mathrm{e}}$ Universidad Antonio de Nebrija, Madrid; ${ }^{\mathrm{f}}$ Universidad Miguel Hernández, Elche; ${ }^{\mathrm{g}}$ Universidad de Salamanca, Salamanca
}

En octubre de 2018, la Asociación Española de Fisioterapeutas en Educación Superior (AEFES) organizó en la sede de la Asociación Española de Fisioterapeutas (AEF) su primera actividad dirigida hacia el colectivo de docentes del Área de Fisioterapia del territorio nacional, y lo hizo centrando la atención sobre uno de los temas que más debate y controversia generan dentro de la universidad española: el sistema de evaluación y acreditación del profesorado universitario, con el objetivo de debatir sobre las repercusiones que dichos sistemas tuvieron en la proyección del Área de Fisioterapia.

Conviene recordar brevemente la trayectoria de los estudios de Fisioterapia dentro de la universidad española, desde su incorporación en el año 1980 con la publicación del Real Decreto 2965/1980 y puesta en marcha de la primera Escuela Universitaria de Fisioterapia en el año 1983 en Valencia (Real Decreto 2702/1983, de 28 de septiembre). En el año 1986se crea el Área de Fisioterapia, a la que se le concedió un carácter específico tres años después, lo que permitió la incorporación de los profesionales de la Fisioterapia a la docencia universitaria; esto supuso el inicio del cuerpo de profesores propios del área a través de las figuras recogidas en la derogada Ley de Reforma Universitaria ${ }^{1}$.

Durante la década de 1990 creció el número de Escuelas Universitarias de Fisioterapia, principalmente de carácter público, y el cuerpo docente de profesores del Área de Fisioterapia con la figura principal del Titular de EscuelaUniversitaria (TEU), a la que se accedía por un sistema de concurso (pruebas públicas ante un tribunal elegido por sorteo entre especialistas en una materia específica). Durante este periodo, una de las principales reivindicaciones del colectivo profesional fue el acceso a un segundo ciclo universitario o una licenciatura en Fisioterapia, que a su vez 
permitiese el acceso a los estudios de tercer ciclo, doctorado y carrera investigadora dentro de la universidad.

A partir del año 2000 se producen dos hechos relevantes en la universidad española que cambian la organización general de la misma. En el año 2001, se aprueba la Ley Orgánica de Ordenación Universitaria y en 2007 se publica el Real Decreto por el que se establece la ordenación delas enseñanzas universitarias oficiales en grado, máster y doctorado (RD 13/93 de 27 de octubre).

La Fisioterapia supo aprovechar esta crisis y se posicionó como una de las primeras titulaciones en adaptarse a los cambios que el sistema de crédito de transferencia euro-peo (ECTS) de Bolonia establece, con la publicación en el año 2004 del Libro Blanco del Título de Grado en Fisioterapia, editado por la ANECA, y que estableció las directrices para la ordenación de los nuevos títulos de Graduado en Fisioterapia, con 240 ECTS durante cuatro años. Posterior-mente se publicó la ORDEN CIN/2135/2008, de 3 de julio, que establecía los requisitos de verificación de los títulos universitarios oficiales que habilitan para la profesión de fisioterapeuta. Como consecuencia, en el año 2008 se produjo la implantación de nuevos títulos universitarios de Graduado en Fisioterapia, y desde ese momento con una nueva estructura de los estudios universitarios de Fisioterapia: grado, máster y doctorado, con un acceso natural de los fisioterapeutas a todos los grados académicos ${ }^{1}$.

A partir de entonces, los centros universitarios trabajan en implantar los nuevos títulos y en generar estudios de máster oficiales, así como programas de doctorado que permitan a sus egresados continuar con su formación en este ámbito científico. En los últimos diez años la Fisioterapia ha experimentado un crecimiento exponencial, lo que supone, según datos aportados por la plataforma QUEDU (consultada en octubre de 2018) del Ministerio de Educación, Cultura y Deporte, que en la actualidad existan 65centros ofertando estudios de Grado en Fisioterapia (42 de ellos de titularidad pública -entre propios y adscritos- y 23 de titularidad privada), 36 estudios de máster (localizados bajo el descriptor «Fisioterapia») y 11 programas de doctorado (6 con «Fisioterapia»; 3 con «Discapacidad» y 2 con «Rehabilitación»).

Todo esto se ha construido en casi cuatro décadas, con un gran esfuerzo por parte de las administraciones y de los profesionales a cargo de la gestión diaria de estos programas: el cuerpo de profesorado del Área de Fisioterapia, sin olvidar el apoyo de la AEF mediante la organización de congresos científicos nacionales e internacionales y la gestión y difusión de la revista científica en español de referencia en fisioterapia.

No obstante, y a pesar de la importancia que ha tenido para el Área de Fisioterapia la nueva legislación, esta ha traído consigo nuevas figuras de profesorado y nuevos sistemas de acceso a la carrera docente a través de los sistemas de acreditación que han tenido un impacto sobre el cuerpo de profesores. Estos sistemas de acreditación se desarrolla-ron con el objetivo de velar por la calidad del profesorado y garantizar que el acceso a la docencia sólo se produzca si se cumplen con unos requerimientos mínimos. Básicamente consisten en una comisión, designada por la Agencia Nacional de Evaluación de la Calidad y Acreditación (ANECA) o por una Agencia equivalente a nivel autonómico - sólo en el caso de las figuras de profesorado contratado y con efectos en su territorio -, formada por profesores de distintas materias - no necesariamente por expertos en la especialidad de quien se presenta -, y que examina el currículum del concursante y le acredita o no como docente en alguna de las diferentes categorías. Los criterios mediante los cuales la comisión adopta sus decisiones son principalmente de naturaleza cuantitativa: número de artículos científicos o libros publicados, comunicaciones en congresos y jornadas, horas de docencia y antigüedad, número de tesis dirigidas, participación en proyectos, etc. En ningún momento se comprueban los conocimientos del concursante ni su grado de preparación para la tarea universitaria ${ }^{2}$. Una vez el con-cursante resulta acreditado, la asignación a la plaza es determinada por las propias universidades a través de sis-temas de concurso público. 
En el caso del Área de Fisioterapia, ninguna de las comisiones de la ANECA en sus diferentes programas - PEP para el profesorado contratado y ACADEMIA para los profesores numerarios: titulares de universidad y catedráticos de universidad - cuenta con profesores del Área de Fisioterapia, por lo que los expedientes están siendo evaluados por profesionales no expertos en el ámbito de la misma. ¿A qué se debe esta falta de representación? La respuesta la podemos inferir si consideramos que para formar parte de estas comisiones existe como requisito el tener reconocidos dos sexenios o tramos de investigación - 3 en el caso de las comisiones que acreditan para catedrático de universidad -, cifra que resulta elevada si se considera que el número medio de sexenios del Área de Fisioterapia es de 0,4, muy por debajo de la media de la rama de conocimiento de ciencias de la salud $(2,5)$ a la que pertenece.

La evaluación de los tramos de investigación o sexenios comenzó en el año 1989 con la publicación del Real Decreto 1086/1989, de 28 de agosto, sobre retribuciones del profesorado universitario y fueron concebidos como un complemento retributivo al profesorado por su actividad investigadora, si bien no se establecieron los procedimientos para su desarrollo hasta el año 1994 (Orden de 2 de diciembre de 1994). Para su evaluación se nombran unas comisiones - en las que tampoco hay representantes del Área de Fisioterapia - y unos criterios que han evolucionado desde unos criterios generales en el año 1994, a unos criterios por ámbitos de conocimiento que se han ido endureciendo en sucesivas convocatorias, imponiendo lo que podríamos llamar «la dictadura del Journal Citation Report $(J C R) »$ : la excesiva dependencia y sobrevaloración de los artículos publicados en revistas incluidas en los listados del JCR en detrimento de otras revistas indexadas en bases similares o superiores como Scopus, que en el caso de las Ciencias de la Salud, contiene indexadas revistas de gran relevancia y prestigio, como la revista Fisioterapia que edita la AEF. Además, acreditar estas aportaciones se ha convertido, de facto, en un requisito sine qua non para obtener el reconocimiento del tramo de investigación. De hecho, sin la aportación de artículos publicados en JCR en los cuartiles que determina la comisión correspondiente, al resto de aportaciones curriculares que pueda tener el candidato (libros, capítulos de libro, revistas indexadas en otras bases de datos, comunicaciones o ponencias presentadas a congresos, participación en grupos de investigación, etc.), se le concede un peso relativo, ponderada y significativamente inferior, que no permite conseguir la acreditación del sexenio.

El Área de Fisioterapia es evaluada a través de los criterios del subámbito de Ciencias de la Salud (B8) que agrupa especialidades sanitarias, junto con otras Áreas como Farmacia y Tecnología Farmacéutica, Farmacología, Medicina Preventiva y Salud Pública, Nutrición y Bromatología, Medicina y Cirugía Animal, Sanidad Animal y Enfermería. Se solicitan los mismos criterios de acreditación de la actividad investigadora en un área en la que hasta hace relativamente poco tiempo (entorno al 2010) era muy difícil o prácticamente imposible desarrollar con plenitud una carrera investigadora; un área en la que en la actualidad un tercio de la plantilla posee el título de doctor o en la que el profesorado funcionario representa el $12 \%$ del total de la plantilla. Dentro de éste, el $46 \%$ son TEU sin sexenio de investigación, el $47 \%$ son Titulares de Universidad, de los que prácticamente la mitad posee un sexenio, un 5\% son Catedráticos de Escuela Universitaria y solamente existen dos Catedráticos de Universidad (según datos aportados por el Sistema Integrado de Información Universitaria - SIIU - del Ministerio de Educación, Cultura y Deporte para el curso académico 2016/17). Se solicitan los mismos criterios para áreas que carecen, porque no les ha dado tiempo a generarlos, de los recursos organizativos básicos para la investigación, de grupos de investigación consolidados y competitivos o de capacidad de captación de recursos de financiación a través de proyectos y programas de doctorado.

Esta situación resulta poco estimulante para un sector de profesorado que, perteneciendo a los cuerpos docentes universitarios, siendo el eje vertebrador de la docencia y la investigación en un departamento y detentando el peso del conocimiento epistemológico e histórico de la disciplina, observa que la actividad que ha venido desarrollando (en su momento evaluada), no les permite continuar con su proyección dentro de la universidad. Un profesorado que progresivamente se va viendo desplazado y segregado de la posibilidad de participar en comisiones evaluadoras, dirigir 
tesis doctorales, figurar como investigador principal en proyectos competitivos, coordinar programas de doctorado o grupos de investigación.

Resulta preocupante además, que la necesaria renovación generacional de estas figuras de profesorado se produzca a expensas de profesorado contratado cuyo mérito principal es fundamentalmente haber publicado artículos en JCR y que en ocasiones desconocen el marco conceptual y profesional de la disciplina, como siempre se le ha exigido a los profesores universitarios a través de la elaboración y defensa de un proyecto docente.

Sin embargo, y a pesar de las dificultades, en los últimos años se ha creado un incipiente tejido investigador que ha permitido mejorar los indicadores de producción científica del Área de Fisioterapia con un aumento progresivo desde el año 2008 del número de tesis doctorales leídas bajo los epígrafes de Fisioterapia y Rehabilitación Médica - Base de Datos TESEO -,con un incremento del número de grupos de investigación en fisioterapia - 39 con datos del año 2017 -, con un aumento del porcentaje de profesores doctores y el número promedio de sexenios ${ }^{3}$ y un incremento del número de publicaciones de autores españoles en el Área de Fisioterapia indexadas en Pubmed/MEDILINE, pasando de menos de 100 en 2012 a cerca de 600 en 2018 (datos no publicados y aproximados).

La instauración de políticas por parte de las autoridades académicas y de las Agencias de Evaluación que considerasen la especificidad y el contexto del Área de Fisioterapia podría estimular el crecimiento del cuerpo de profesores del Área de Fisioterapia y su productividad científica. Estas se podrían sintetizar en:

- Revisión de los criterios de evaluación en los diferentes programas de la ANECA, considerando los factores con-textuales de la disciplina. El profesorado del Área de Fisioterapia en España investiga, pero los criterios actuales con los que se evalúa esta actividad hacen que no se visibilice adecuadamente.

- Incorporación de la valoración de revistas indexadas en otras bases de datos como SCOPUS, ya que esta base es a nivel científico la que mayor cobertura tiene de las Ciencias de la Salud, incluyendo el 100\% de PUBMED, y que además tiene incluida la revista Fisioterapia, principal vía de difusión de los artículos de investigación de los fisioterapeutas españoles.

- Mejora de la sensibilidad de las comisiones de evaluación por medio de la incorporación de profesorado perteneciente al Área de Fisioterapia.

La AEF-ES está comprometida con todos estos objetivos y propuestas, y con seguir trabajando en sinergia con las instituciones académicas, profesionales y científicas de la fisioterapia española en los diferentes retos académicos y científicos a los que se enfrenta la disciplina.

\section{Bibliografía}

1. Fernández Cervantes R, Souto Gestal A. Competencias del fisioterapeuta y su capacidad facultativa. En: VII Jornadas Nacionales y IV Internacionales de Educación en Fisioterapia. Ponferrada: UNILEON; 2011.

2. De Carreras F. Tres problemas de la Universidad. El país Opinión. 15 de diciembre de 2014.

3. González Doniz L, Souto Camba S, Gómez Conesa A, Suárez Serrano C, González Cabanach R. Características, tipificación y análisis de la producción de tesis doctorales en el área de Fisioterapia. En: XIV Foro Internacional sobre Evaluación de la Calidad de la Educación Superior y de la Investigación. Granada: Asociación Española de Psicología Conductual; 2017. p. 242. 